\title{
Nonsurgical and surgical periodontal therapy in single-rooted teeth
}

\author{
Ti-Sun Kim • Aniela Schenk • Diana Lungeanu • \\ Peter Reitmeir · Peter Eickholz
}

Received: 7 April 2007 / Accepted: 16 July 2007 / Published online: 10 August 2007

(C) Springer-Verlag 2007

\begin{abstract}
The purpose of this study was to compare the effect of tooth related and patient related factors on the success of non-surgical and surgical periodontal therapy. In 41 patients (22 female) with untreated and/or recurrent periodontitis, no therapy, scaling and root planing (SRP), or access flap (AF) were assigned according to probing pocket depth (PPD). PPD and vertical relative attachment level (RAL-V) were obtained initially, 3 and 6 months after therapy. Baseline data were compared according to therapy, jaw, tooth type, and site. Factors influencing clinical parameters were identified using multilevel analyses.
\end{abstract}

T.-S. Kim • A. Schenk

Section of Periodontology, Department of Conservative Dentistry, Clinic for Oral, Dental, and Maxillofacial Diseases,

University Hospital Heidelberg,

69120 Heidelberg, Germany

D. Lungeanu

Medical Informatics and Biostatistics Department,

University of Medicine and Pharmacy Timisoara,

300041 Timisoara, Romania

\section{P. Reitmeir}

Institute of Health Economics and Health Care Management, GSF-National Research Center for Environment and Health, 85758 Neuherberg, Germany

\section{P. Eickholz}

Department of Periodontology, Center for Dental, Oral, and Maxillofacial Medicine, University Hospital Frankfurt, 60590 Frankfurt, Germany

\section{P. Eickholz $(\square)$}

Poliklinik für Parodontologie, Zentrum der Zahn-, Mund- und Kieferheilkunde (Carolinum), Klinikum der Johann Wolfgang Goethe Universität, Frankfurt/Main,

Theodor-Stern-Kai 7,

60590 Frankfurt/Main, Germany

e-mail: eickholz@med.uni-frankfurt.de
Baseline PPDs were deeper interproximally, in the maxilla and at premolars compared to buccal/oral sites, mandibular, and anterior teeth. At 6 months, PPD reduction and RAL-V gain were significantly greater at sites receiving SRP and $\mathrm{AF}$ as compared to untreated sites $(p<0.001)$. PPD reduction and RAL-V gain were significantly less $(p<$ $0.005)$ in smokers as compared to nosmokers and at interproximal sites $(p<0.0001)$ as compared to buccal/oral sites. RAL-V gain was less in aggressive periodontitis, and PPD reduction was less in the maxilla $(p<0.001)$. In sites with greater bone loss and infrabony defects, a poorer response was observed regarding RAL-V gain or PPD reduction, respectively. The conclusions of the study are the following: (1) Nonsurgical and surgical periodontal therapies are effective in single-rooted teeth; (2) severe interproximal bone loss and infrabony defects deteriorate clinical results; and (3) there seem to be more defectassociated (tooth, site) factors influencing treatment outcome than patient-associated factors.

Keywords Periodontal therapy .

Surgical/nonsurgical therapy · Single-rooted teeth .

Clinical trial $\cdot$ Smoking

\section{Introduction}

The major factor in the etiology of periodontitis is the dental biofilm, i.e., bacterial plaque. Thus, the aim of antiinfective periodontal therapy is the reduction in supra- and subgingival microbial plaque to resolve periodontal inflammation. Subgingival scaling and root planing (SRP) may result in resolution of inflammation, reduced probing pocket depths (PPD), and clinical attachment gain [1, 2, 16]. However, nonsurgical periodontal therapy, i.e., SRP, is 
not found to be successful at all treated sites. Although not all factors influencing failure of nonsurgical therapy are known, deep periodontal pockets [3, 21, 25, 28] and furcation involvement $[6,9,23]$ are some of them. Recent meta-analyses have shown the efficacy of nonsurgical therapy in moderate and deep periodontal pockets. In deep pockets, access flap (AF) techniques result in better reduction in PPD and clinical attachment gain [14, 15]. However, these meta-analyses like many other studies on this issue before $[3,21,25,28]$ use mean values and are not able to reflect the benefit of a particular technique at teeth with certain defects, e.g., infrabony pockets. However, calculation of a mean value, e.g., for PPD reduction, across a patient in fact levels out tooth-related characteristics. Even if separate strata for different baseline PPD are considered, tooth-related factors other than baseline PPD are leveled out also. This may lead to quite similar mean values for nonsurgical and surgical therapy for a patient or across a whole sample because tooth-related differences between both approaches because of different amounts or types of bone loss, jaw, etc. are leveled out. More recent statistical methods such as the multilevel modeling are considering single-tooth or even site characteristics while still regarding the individual patient as a statistical unit. In a structured review of 2002, it was explicitly stated that the included studies showed similar results for nonsurgical and surgical therapy after 1 year. However, insufficient studies are available to evaluate the various treatment procedures, e.g., in angular defects. Further, it is stated that subject characteristics and their possible effect on treatment outcome was not addressed in the studies available at that time [14]. Thus, there is a need for more information on the effect of patient and tooth-related factors on nonsurgical and surgical periodontal therapy. Well known hypotheses and the use of these more recent statistical methods may lead to new information.

Clinical measurements in posterior are less reproducible than in anterior teeth [7]. Furcation involvement is a known factor to influence treatment outcome of nonsurgical and surgical therapy. To provide a high level of reproducibility of clinical measurements and to exclude a already known influencing factor, only single-rooted teeth were considered in this study.

The hypothesis behind this study is that different toothrelated factors (e.g., tooth type, site, plaque, residual inflammation, horizontal/vertical bone loss) and patientrelated factors (e.g., age, sex, diagnosis, smoking) determine the initial outcome of nonsurgical and surgical periodontal therapy in single-rooted teeth.

Thus, the aim of the present study was to identify patient and site factors that determine the initial outcome of nonsurgical and surgical periodontal therapy in singlerooted teeth using more recent statistical methods.

\section{Materials and methods}

\section{Patients}

The study group consisted of 41 patients (22 female) suffering from moderate to severe periodontal disease. These patients had been scheduled for periodontal treatment within a randomized placebo-controlled clinical trial evaluating the effect of a subgingivally delivered $14 \%$ doxycycline gel at the Section of Periodontology, Department of Conservative Dentistry, Clinic for Oral, Dental and Maxillofacial Diseases, University Hospital Heidelberg. Each patient had to have three single-rooted teeth that qualified as test teeth for the doxycycline study; that is, only three teeth per patient were included into this study [8]. Inclusion criteria were at least 23 years of age, untreated moderate to severe periodontal disease or recurrent periodontitis without periodontal surgery at least for the last 24 months, effective individual oral hygiene (plaque control record $<35 \%$ ) [24], and written informed consent. Exclusion criteria were local and/or systemic antibiotic therapy within the last 6 months before the baseline examination of the study, known adverse reactions to any component of the test agent, anticoagulative therapy, liver, kidney, gastroenteral diseases, pregnancy, and women who were lactating. All patients were asked about current and past cigarette consumption. A patient was classified as a current smoker if he smoked one or more cigarettes per day and as a former smoker if he had quit smoking for at least 5 years. Patients who had quit smoking within the last 5 years were classified current smokers. No more than $50 \%$ of current smokers were allowed in the sample. All patients were classified as chronic or aggressive periodontitis according to the following criteria:

- Aggressive periodontitis: Patient is clinically healthy; that is, systemic diseases predisposing for periodontitis are not known; radiographic bone loss greater than or equal to $50 \%$ in at least two different teeth and age less that or equal to 35 years at time of diagnosis.

- Moderate and severe chronic periodontitis: attachment loss greater than or equal to $3 \mathrm{~mm}$ and age greater than 35 years [19].

The study was approved by the Institutional Review Boards for Human Studies of the University of Heidelberg.

\section{Clinical examination}

After completion of initial periodontal treatment including oral hygiene instruction and supragingival scaling, PPD were measured at four sites of every tooth. If a patient fulfilled the inclusion criteria, the study protocol, risks, benefits, and procedures were explained, and informed 
written consent was obtained. One week before test therapy, the Gingival Index (GI) and Plaque Index (PII) [22] were assessed at six sites (mesiobuccal, midbuccal, distobuccal, distolingual, midlingual, mesiolingual) of every incisor, canine, and premolar. PPD and vertical relative attachment levels (RAL-V) were measured to the nearest $0.5 \mathrm{~mm}$ using a straight periodontal probe (PCPUNC 15, Hu Friedy, Chicago, IL) at six sites per tooth. A reference splint gave the location of probing by notches and served as reference for the RAL-V measurements. Bleeding on probing (BOP) was recorded approximately $30 \mathrm{~s}$ after probing. After the first course of measurements, all PPD and RAL-V measurements were repeated. The means of the pairs of double measurements were used for analysis. If a pair of PPD double measurements showed a difference greater than $2 \mathrm{~mm}$, a third measurement was performed at the respective site (option 3). Then, the mean of the pair of the closer measurements was taken for analysis at the respective site. All clinical measurements before therapy were performed by one examiner (Eickholz) who also performed active periodontal therapy (subgingival debridement and surgery) in all patients. Within each patient, three test teeth with $\mathrm{PPD}=5 \mathrm{~mm}$ and $\mathrm{BOP}$ or $\mathrm{PPD} \geq 6 \mathrm{~mm}$ were selected for the doxycycline study. These teeth were excluded from this examination. Three and 6 months after therapy, the clinical examinations were repeated in the same manner by a second examiner (Kim) who did not have any information about baseline data and the therapy [8].

\section{Radiographic examination and evaluation}

Before therapy, in 36 patients, full-mouth sets of periapical radiographs (Ektaspeed plus; Kodac, Rochester, NY) were obtained in XCP technique (XCP, Kentzler \& Kaschner Dental, Ellwangen/Jagst, Germany). Intraoral size 0 (maxillary second incisors and mandibular anteriors) and 2 (all other regions) dental films were exposed to an X-ray source (Heliodent DS ${ }^{\circledR}, 60$ kV, 7 mA, Sirona, Bensheim, Germany) and developed under standardized conditions (XR 24 Nova $^{\circledR}$, Dürr Dental, Bietigheim-Bissingen, Germany).

All radiographs were viewed in a darkened room using a radiograph screen (67-0420, Dentsply Rinn, Elgin, IL). Each interproximal surface of single-rooted teeth was classified for the type of bone loss (horizontal/vertical). Further, the prevalence of a double contour of the root was noted that was taken as indicative for a mesial or distal groove. Relative bone loss in percent was assessed at two sites per tooth (mesial and distal) using a Schei ruler [27]. This ruler consisted of six straight lines that divert in the same angle. For each site, the basic line of the Schei ruler was placed to the cementoenamel junction (CEJ) of the respective tooth parallel to the occlusal plane. Then, the ruler was moved until the sixth line was placed tangentially to the apex. Finally, the alveolar crest was located. Location between the two coronal lines meant bone loss up to $20 \%$ of root length. Location between the second and third line meant between 20 and $40 \%$ bone loss and so forth. If the CEJ was destroyed or overlapped by interproximal restorations, the restoration margin was used as reference. If the alveolar crest could not be determined because of overlapping of adjacent teeth, the interproximal site was classified as "cannot be assessed." If an interproximal surface showed a double contour, this was classified as an interproximal root groove. All radiographic assessments were performed by an examiner who was blinded to the clinical measurements and therapy assignments (Schenk).

\section{Therapy}

Teeth exhibiting $\mathrm{PPD}<3 \mathrm{~mm}$ were treated neither by subgingival SRP nor by AF [26]. SRP was performed when $P P D \geq 3$ and $\leq 6 \mathrm{~mm}$ were present. If periodontal surgery was necessary at teeth not adjacent to test teeth, this was performed 1 week after SRP: AF was chosen for teeth exhibiting PPD $>6 \mathrm{~mm}$. However, SRP was performed at all test teeth (not included in this analysis) and the respective neighboring teeth under local anesthesia with hand instruments even for baseline PPD $>6 \mathrm{~mm}$. Thus, also some sites with PPD $>6 \mathrm{~mm}$ were treated exclusively by SRP. Further, the decision to perform AF was taken per tooth and not per site. Thus, also sites with $\mathrm{PPD} \leq 6 \mathrm{~mm}$ were treated by AF if the respective tooth exhibited $\mathrm{PPD}>6 \mathrm{~mm}$ at one site. Thereby, effectiveness of SRP and AF maybe analyzed across the limits of indications as the ranges of PPD for the three treatment groups in Table 4 demonstrate. Maintenance including oral hygiene instruction and professional tooth cleaning was done 3 months after test therapy.

Statistical analysis

\section{Data handling}

All data were entered into a personal computer program (Systat $^{\mathrm{TM}}$ for Windows version 10, Systat, Evanston, IL; Schenk). Descriptive statistics were then calculated using another program (SPSSTM, version 10.0.1, SPSS, Chicago IL) by an independent statistician (Lungeanu). Multilevel analyses were modeled by another independent statistician (Reitmeir) using a third program (SAS ${ }^{\circledR}$ version 6.12, SAS Institute, Cary, NC).

\section{Reproducibility}

The replicate PPD and RAL assessments for all teeth were used to estimate the intraexaminer reproducibility. The intraexaminer reproducibility of PPD and RAL measure- 
ments was expressed as the standard deviation of single measurements [4].

\section{Treatment comparison}

The individual patient was considered as a statistical unit in this analysis. The main outcome variable for the comparison of the therapeutic effects of no further therapy, SRP, or AF was chosen to be the change of PPD. RAL-V was considered as a secondary endpoint. All other clinical parameters (GI, PII) were control variables.

Only sites were included that could be assessed at all three examinations. Additionally, baseline clinical parameters were calculated separately according to therapy (no therapy, SRP, AF), jaw, tooth type (anterior/premolar), and site (interproximal/buccal and oral) and compared using multilevel methodology $[10,11]$. A probability $p<0.05$ was accepted for a difference to be statistically significant. Because of multiple testing, a Bonferroni correction was performed: We tested 24 independent hypotheses on our set of data. Thus, the statistical significance level that should be used for each hypothesis separately is 1 of 24 times what it would be if only one hypothesis were tested, i.e., $p=$ 0.002. For all analyses, the basic level "site" was nested in the upper level "tooth," which itself was nested in the next upper level "patient," and patient effects on the outcome were assumed to be random. This technique allows the identification of single-tooth effects or parameters while still considering the individual patient as a statistical unit and the dependencies of site and tooth data within a patient $[10,11]$. Multilevel regressions were calculated including only clinical parameters providing six measurements per tooth first (site level: $n=4,146$; patient level: $n=40$ ). Further analyses were performed that included also radiographic parameters. Radiographic parameters were only available for those patients contributing full-mouth radiographic examinations and could be related only to interproximal sites (mesiobuccal, mesiolingual, distolingual, mesiolingual), i.e., four sites per tooth (site level: $n=1,792$; patient level: $n=36$ ).

Table 1 Study population as related to age, sex, smoking habit, and diagnosis

\begin{tabular}{llll}
\hline Variable & & Number $(n=41)$ & Percent \\
\hline Age & $45.9 \pm 10.9$ & & \\
Sex & Female & 22 & 53.7 \\
Smoking & Active & 19 & 46.3 \\
& Former & 12 & 29.3 \\
& Never & 10 & 24.4 \\
Diagnosis & Aggressive & 8 & 19.5 \\
\hline
\end{tabular}

Table 2 Intraexaminer reproducibility at baseline, 3, and 6 months examination

\begin{tabular}{llll}
\hline Examination & Measurements $(n)$ & $s(\mathrm{RAL}-\mathrm{V})$ & $s(\mathrm{PPD})$ \\
\hline Baseline & 8,400 & 0.49 & 0.49 \\
3 months & 8,376 & 0.44 & 0.39 \\
6 months & 8,292 & 0.53 & 0.39
\end{tabular}

$s$ Standard deviations of a single measurement

To identify factors influencing the treatment result, change of RAL-V and PPD were analyzed by application of a multilevel regression model using backward selection. Models were fitted for the dependent variables (1) RAL-V change after 6 months and (2) PPD change after 6 months including the following independent variables: (1) clinical: age, sex, actual smoking (yes/no), pack years, diagnosis (aggressive/chronic periodontitis), jaw (maxilla/mandible), site (interproximal/buccal or oral), tooth type (anterior/ premolar), therapy (none/SRP/AF), and PII and GI at baseline, (2) radiographic: interproximal bone loss in percent, infrabony defect, and double contour of the root. For the analysis including radiographic parameters, the variable "site" (interproximal/buccal and oral) dropped out because only interproximal sites remained in the analysis. A probability $p<0.15$ was required for parameters to be kept within the models.

\section{Results}

All 41 patients contributing 700 single-rooted teeth with 4,200 sites finished the 3-month re-examination. The patient characteristics (age, diagnosis, smoking status) are given in Table 1. From baseline to the 3-month examination, two single-rooted teeth were lost: one to an acute periapical periodontitis and another because of prosthodontic planning. After the 3-month re-examination, one patient left the study. Only sites were included into the analysis, which could be assessed at all three examinations: $n=4,146$ in 40 patients.

Table 3 Clinical parameters at baseline, 3 and 6 months after therapy (mean \pm standard deviation)

\begin{tabular}{llll}
\hline & Baseline & 3 months & 6 months \\
\hline Sites & 4,146 & 4,146 & 4,146 \\
RAL-V (mm) & $5.12 \pm 2.20$ & $4.87 \pm 1.87$ & $4.92 \pm 1.84$ \\
PPD (mm) & $3.25 \pm 1.83$ & $2.67 \pm 1.19$ & $2.67 \pm 1.20$ \\
GI & $0.78 \pm 0.97$ & $0.51 \pm 0.86$ & $0.51 \pm 0.86$ \\
PII & $0.16 \pm 0.51$ & $0.32 \pm 0.70$ & $0.37 \pm 0.74$ \\
\hline
\end{tabular}


Table 4 Clinical parameters at baseline as related to therapy (mean \pm standard deviation)

\begin{tabular}{|c|c|c|c|c|c|c|}
\hline \multirow[t]{2}{*}{ Sites } & \multicolumn{2}{|l|}{ No therapy } & \multicolumn{2}{|l|}{ SRP } & \multicolumn{2}{|l|}{$\mathrm{AF}$} \\
\hline & 1,440 & Range & 2,478 & Range & 198 & Range \\
\hline RAL-V/mm & $4.35 \pm 1.82 *$ & $1.25-19.00$ & $5.55 \pm 2.30$ & $1.75-18.00$ & $5.39 \pm 2.02$ & $2.25-12.00$ \\
\hline $\mathrm{PPD} / \mathrm{mm}$ & $2.45 \pm 1.13^{*}$ & $0.25-2.75$ & $3.66 \pm 1.98$ & $0.50-12.25$ & $3.98 \pm 2.02$ & $1.00-10.50$ \\
\hline GI & $0.48 \pm 0.84^{*}$ & & $0.94 \pm 1.00$ & & $1.02 \pm 0.98$ & \\
\hline PII & $0.15 \pm 0.49$ & & $0.16 \pm 0.53$ & & $0.14 \pm 0.49$ & \\
\hline
\end{tabular}

$S R P$ Scaling and root planning, $A F$ access flap

*Statistically significantly different from SRP and AF with $p<0.001$

\section{Reproducibility}

The results of the intraexaminer reproducibility of PPD and RAL measurements as standard deviation of single measurement for each examination time point are given in Table 2.

\section{Baseline data}

The mean clinical parameters (RAL-V, PPD, PII, GI) at baseline over all sites are given in Table 3 . The respective data as related to therapy (no therapy, SRP, AF) are given in Table 4. Whereas no statistically significant differences for PII were observed between the groups, RAL-V, PPD, and GI of the SRP and AF sites revealed to be significantly ( $p<$ 0.001 ) greater as compared to untreated sites (Table 4). Clinical parameters as related to jaw, tooth type, and site are given in Table 5. Baseline RAL-V and PPD were statistically significantly $(p<0.001)$ greater in the maxilla than in the mandible, whereas for PII, it was vice versa (Table 5). For tooth type, just baseline PPD was statistically significantly $(p<0.001)$ different between premolars and anteriors with deeper pockets at the premolars (Table 5). All baseline clinical parameters were observed to be statistically significantly $(p<0.001)$ greater at the interproximal than at the buccal and oral sites (Table 5).
Treatment effect

The healing phase passed uneventfully for all patients. Clinical parameters as related to therapy after 3 and 6 months are given in Table 6, and their change after 3 and 6 months is given in Table 7. Whereas in untreated sites, the study failed to observe any significant change, $\mathrm{SRP}$ and AF resulted in PPD reduction and PAL-V gain after 3 and 6 months $(p<0.001$; Table 7).

\section{Prognostic factors}

RAL-V gain was less favorable in current smokers, in aggressive periodontitis, and at interproximal sites (Table 8). PPD reduction was poorer in current smokers, at premolars, in the maxilla, and at interproximal sites (Table 9). Whereas baseline PPD was related to better PPD reduction and RAL$\mathrm{V}$ gain $(p<0.0001)$, baseline RAL-V was related to better RAL-V gain but to less favorable PPD reduction (Tables 8 and 9). Baseline PII was associated with poorer PPD reduction $(p<0.15$; Table 9). AF and SRP $(p=0.0114)$ were associated with better PPD reduction (Table 9).

Taking radiographic parameters into consideration, current smoking resulted in less favorable and baseline PPD in better RAL-V gain and PPD reduction, respectively (Tables 10 and 11). Further, tooth type (premolar) and

Table 5 Clinical parameters at baseline as related to jaw (maxilla/mandible), tooth type (anterior/premolar), and site (interproximal/buccal and oral) (mean \pm standard deviation)

\begin{tabular}{|c|c|c|c|c|c|c|}
\hline & Maxilla & mandible & premolar & anterior & interproximal & buccal/oral \\
\hline Sites & 1,926 & 2,220 & 1,590 & 2,566 & 2,764 & 1,382 \\
\hline RAL-V/mm & $5.24 \pm 2.14 *$ & $5.00 \pm 2.26$ & $5.19 \pm 2.20 *$ & $5.07 \pm 2.22$ & $5.37 \pm 2.26^{*}$ & $4.60 \pm 2.01$ \\
\hline $\mathrm{PPD} / \mathrm{mm}$ & $3.55 \pm 1.88^{*}$ & $3.00 \pm 1.78$ & $3.47 \pm 1.88^{*}$ & $3.12 \pm 1.81$ & $3.67 \pm 1.82 *$ & $2.43 \pm 1.59$ \\
\hline GI & $0.80 \pm 0.98 *$ & $0.76 \pm 0.96$ & $0.83 \pm 0.97 *$ & $0.75 \pm 0.96$ & $0.89 \pm 0.98^{*}$ & $0.56 \pm 0.89$ \\
\hline PII & $0.11 \pm 0.43^{*}$ & $0.19 \pm 0.57$ & $0.14 \pm 0.48 *$ & $0.17 \pm 0.53$ & $0.18 \pm 0.54 *$ & $0.11 \pm 0.44$ \\
\hline
\end{tabular}

*Statistically significantly different with $p<0.001$ 
Table 6 Clinical parameters at 3 and 6 months after therapy as related to therapy (mean \pm standard deviation)

\begin{tabular}{|c|c|c|c|c|c|c|}
\hline & \multicolumn{3}{|c|}{3 months after therapy } & \multicolumn{3}{|c|}{6 months after therapy } \\
\hline & No therapy & SRP & $\mathrm{AF}$ & No therapy & SRP & $\mathrm{AF}$ \\
\hline Sites & 1,440 & 2,478 & 198 & 1,440 & 2,478 & 198 \\
\hline RAL-V (mm) & $4.37 \pm 1.76$ & $5.13 \pm 1.90$ & $5.14 \pm 1.64$ & $4.47 \pm 1.76$ & $5.17 \pm 1.85$ & $5.11 \pm 1.61$ \\
\hline PPD (mm) & $2.33 \pm 0.96$ & $2.84 \pm 1.25$ & $3.09 \pm 1.34$ & $2.35 \pm 1.05$ & $2.83 \pm 1.24$ & $2.99 \pm 1.27$ \\
\hline GI & $0.36 \pm 0.76$ & $0.58 \pm 0.90$ & $0.69 \pm 0.94$ & $0.36 \pm 0.76$ & $0.58 \pm 0.90$ & $0.65 \pm 0.93$ \\
\hline PII & $0.22 \pm 0.56$ & $0.36 \pm 0.74$ & $0.52 \pm 0.89$ & $0.31 \pm 0.68$ & $0.41 \pm 0.78$ & $0.27 \pm 0.65$ \\
\hline
\end{tabular}

$S R P$ Scaling and root planing, $A F$ access flap

aggressive periodontitis were associated with poorer RAL$\mathrm{V}$ gains (Table 10). Location in the maxilla and baseline PII resulted in less favorable PPD reduction (Table 11). Generally, sites with greater interproximal bone loss demonstrated poorer RAL-V gain $(p=0.0001)$, and sites with infrabony defects were associated with inferior PPD reduction $(p<0.0001$; Tables 10 and 11).

\section{Discussion}

\section{Study population}

Patients treated in this study ranged from 23 to 71 years of age with chronic and aggressive periodontitis. Original measurements were performed in the setting of a randomized placebo-controlled clinical trial evaluating the effect of a subgingivally delivered $14 \%$ doxycycline gel at the Section of Periodontology, Department of Conservative Dentistry, Clinic for Oral, Dental and Maxillofacial Diseases, University Hospital Heidelberg. Each patient had to provide three single-rooted teeth that qualified as test teeth for the doxycycline study. To blind the examiner for the test teeth, not only those but all single-rooted teeth were assessed in all patients [8]. Despite the test teeth and their direct neighbors, all single-rooted teeth were treated according to the following criteria: Teeth exhibiting $\mathrm{PPD}<$ $3 \mathrm{~mm}$ were treated neither by subgingival SRP nor by AF. SRP was performed when $P P D \geq 3 \mathrm{~mm}$ was present. AF was chosen for teeth exhibiting PPD $>6 \mathrm{~mm}$ [21]. However, teeth directly adjacent to test teeth were treated only by SRP irrespective of baseline PPD. Thus, also some sites with PPD $>6 \mathrm{~mm}$ were treated exclusively by SRP. Further, the decision to perform AF was taken per tooth and not per site. Thus, also sites with PPD $\leq 6 \mathrm{~mm}$ were treated by AF, if they were located at a tooth with one or more sites with PPD $>6 \mathrm{~mm}$. Thereby, effectiveness of SRP and AF may be analyzed across the limits of indications as the ranges of PPD for the three treatment groups in Table 4 demonstrate.

How could we exclude that the topically delivered doxycycline had any influence on the treatment results of the sites that were evaluated in this study? The subgingivally delivered agent or vehicle control may be partially absorbed and thereby have systemic effects at the other test teeth. A pharmacokinetic study had found doxycycline only in the blood of 1 of 16 patients [18]. Thus, a systemic additional effect on the other teeth is quite unlikely. It has to be kept in mind that in the sample investigated in this study, only one tooth of the whole dentition per patient received the active agent, i.e., doxycycline [8]. Further, amounts of the subgingivally delivered substance may leave the pocket via sulcus fluid and affect the other test teeth. Split-mouth

Table 7 Change of clinical parameters at 3 and 6 months after therapy as related to therapy (mean \pm standard deviation)

\begin{tabular}{|c|c|c|c|c|c|c|}
\hline & \multicolumn{3}{|c|}{3 months after therapy } & \multicolumn{3}{|c|}{6 months after therapy } \\
\hline & No therapy & SRP & $\mathrm{AF}$ & No therapy & SRP & $\mathrm{AF}$ \\
\hline Sites & 1,440 & 2,478 & 198 & 1,440 & 2,478 & 198 \\
\hline RAL-V (mm) & $0.03 \pm 0.74$ & $0.41 \pm 1.11 *$ & $0.26 \pm 1.06^{*}$ & $0.12 \pm 0.94$ & $0.38 \pm 1.20 *$ & $0.28 \pm 1.18^{*}$ \\
\hline PPD (mm) & $-0.11 \pm 0.72$ & $-0.82 \pm 1.33^{*}$ & $-0.89 \pm 1.25^{*}$ & $0.09 \pm 0.87$ & $-0.82 \pm 1.40 *$ & $-0.99 \pm 1.41^{*}$ \\
\hline GI & $-0.13 \pm 0.97$ & $-0.36 \pm 1.14 *$ & $-0.33 \pm 1.14^{*}$ & $-0.13 \pm 1.03$ & $-0.36 \pm 1.18^{*}$ & $-0.36 \pm 1.44^{*}$ \\
\hline PII & $0.08 \pm 0.69$ & $0.20 \pm 0.82 *$ & $0.37 \pm 0.97 *$ & $0.16 \pm 0.75$ & $0.25 \pm 0.87^{*}$ & $0.13 \pm 0.68^{*}$ \\
\hline
\end{tabular}

SRP Scaling and root planing, $A F$ access flap

*Statistically significantly different from no therapy with $p<0.001$ 
Table 8 Backward multilevel linear regression analysis — dependent variable: RAL-V reduction 6 months after therapy, $n=40$ patients, 4,146 sites

\begin{tabular}{|c|c|c|c|c|c|}
\hline & Estimate & SE & $d f$ & $F$ value & $p$ value \\
\hline Intercept & -1.8776 & 0.1068 & 37 & & \\
\hline Current smoking & -0.2875 & 0.1106 & 3,427 & 6.76 & 0.0094 \\
\hline Site (interproximal) & -0.2341 & 0.0277 & 3,427 & 71.28 & $<0.0001$ \\
\hline Aggressive periodontitis & -0.2903 & 0.1308 & 3,427 & 4.93 & 0.0265 \\
\hline Baseline PPD & 0.2746 & 0.0158 & 3,427 & 303.72 & $<0.0001$ \\
\hline Baseline RAL-V & 0.1832 & 0.0141 & 3,427 & 169.61 & $<0.0001$ \\
\hline
\end{tabular}

studies designed to compare active agent and vehicle of topical antibiotics have made the provision that teeth selected as test sites should be separated by at least one tooth $[8,12]$, minimizing the interaction between therapy modes. In a pharmacokinetic study from control sites, much smaller amounts of doxycycline could be detected in the gingival crevicular fluid than from another site that had received the particular doxycycline gel subgingivally in the same dentition [20]. Thus, it is unlikely that the topical doxycycline had any additional effect on the treatment results of all teeth but the test teeth.

\section{Treatment effect}

The results observed in this study after SRP are in agreement with observations reported by other authors 3 and 6 months after nonsurgical debridement of singlerooted teeth $[2,6]$. Our PPD reductions and RAL-V gains achieved 6 months after therapy confirm data by a metaanalysis on nonsurgical vs surgical periodontal therapy that reports PPD reduction of $1.07 \mathrm{~mm}$ and vertical attachment (PAL-V) gains of $0.6 \mathrm{~mm}$ at sites with baseline PPD 4$6 \mathrm{~mm} 6$ months after SRP. However, PPD reductions 6 months after AF for mean baseline PPD of $5.4 \mathrm{~mm}$ were $1.0 \mathrm{~mm}$ which is less than the meta analysis lists for baseline PPD 4-6 mm: PPD reduction $1.37 \mathrm{~mm}$. PAL-V gain after AF was $0.3 \mathrm{~mm}$ which comes quite close to the $0.31 \mathrm{~mm}$ reported by the meta analysis [15]. Serino et al. report results 12 months after treatment [28]. For baseline PPD 4-5 mm they report PAL-V gains of $0.31 \mathrm{~mm}$ and PPD reduction of $1.1 \mathrm{~mm}$ after non-surgical as well as $0.21 \mathrm{~mm}$ and $1.6 \mathrm{~mm}$ after surgical therapy. This is quite similar to the results of this study regarding PAL-V gain and PPD reduction after SRP. However, the PPD reduction after surgical therapy is more favorable than ours [28].

Prognostic factors

Current smoking was generally associated with less favorable PPD reduction and RAL-V gain in all models. Smoking is the most significant external risk factor for periodontitis $[5,13,29]$ and is known to deteriorate the results of periodontal therapy in general $[7,30]$. However, smoking was the only patient-associated factor that was kept in all models. The diagnosis of aggressive periodontitis was associated with less favorable RAL-V gains, whereas it was not kept in the models to explain PPD reduction. It is interesting to note that the other patientassociated factors, i.e., age and sex, were kept in neither model. This confirms observations of D'Aiuto et al. [5].

It is interesting to note that the diagnosis of aggressive periodontitis affected RAL-V gains but not PPD reduction.

Table 9 Backward multilevel linear regression analysis — dependent variable: PPD reduction 6 months after therapy, $n=40$ patients, 4,146 sites

\begin{tabular}{|c|c|c|c|c|c|}
\hline & Estimate & SE & $d f$ & $F$ value & $p$ value \\
\hline Intercept & -1.6487 & 0.1037 & 38 & & \\
\hline Current smoking & -0.3312 & 0.1055 & 3,451 & 9.86 & 0.0017 \\
\hline Jaw (maxilla) & -0.1299 & 0.0324 & 3,451 & 16.05 & $<0.0001$ \\
\hline Site (interproximal) & -0.3664 & 0.0262 & 3,451 & 195.10 & $<0.0001$ \\
\hline Tooth type (premolar) & -0.0501 & 0.0338 & 3,451 & 2.20 & 0.1381 \\
\hline Baseline PPD & 0.6162 & 0.0146 & 3,451 & 1774.31 & $<0.0001$ \\
\hline Baseline RAL-V & -0.0354 & 0.0128 & 3,451 & 7.63 & 0.0058 \\
\hline Baseline Plaque Index & -0.0368 & 0.0244 & 3,451 & 2.27 & 0.1318 \\
\hline Scaling and root planing & 0.1171 & 0.0392 & 3,451 & 4.48 & 0.0114 \\
\hline Access flap & 0.0997 & 0.0861 & 3,451 & 4.48 & 0.0114 \\
\hline
\end{tabular}


Table 10 Multilevel linear regression analysis - dependent variable: RAL-V reduction 6 months after therapy, $n=36$ patients, 1,796 sites

\begin{tabular}{|c|c|c|c|c|c|}
\hline & Estimate & SE & $d f$ & $F$ value & $p$-value \\
\hline Intercept & -1.8290 & 0.1385 & 33 & & \\
\hline Current smoking & -0.3758 & 0.1334 & 1,313 & 7.94 & 0.0049 \\
\hline Tooth type (premolar) & -0.0763 & 0.0502 & 1,313 & 2.31 & 0.1292 \\
\hline Aggressive periodontitis & -0.3311 & 0.1635 & 1,313 & 4.10 & 0.0431 \\
\hline Baseline PPD & 0.2371 & 0.0256 & 1,313 & 85.86 & $<0.0001$ \\
\hline Baseline RAL-V & 0.2637 & 0.0242 & 1,313 & 118.36 & $<0.0001$ \\
\hline Bone loss in \% & -0.0115 & 0.0016 & 1,313 & 50.58 & $<0.0001$ \\
\hline
\end{tabular}

It seems that patients suffering from aggressive periodontitis have a higher risk for recessions than patients with chronic periodontitis.

Several tooth- and site-associated factors were identified by multilevel analysis to influence clinical outcomes. Generally, baseline PPD was related to better PPD reduction and RAL-V gain $(p<0.0001)$. PPD reduction and RAL-V gain was less pronounced in the premolars and at interproximal sites $(p<0.15)$, and PPD reduction was less pronounced in the maxilla $(p<0.001)$. These results are plausible because compared to anterior teeth, premolars are less accessible and interproximal sites are more difficult to debride than oral and lingual sites. Less favorable PPD reduction in premolars than in incisors was also recently reported by other authors [5]. However, D'Aiuto et al. observed more favorable PPD reduction at interproximal sites, whereas this study observed the contrary. The multilevel analysis performed in this study was adjusted for baseline PPD. Baseline pockets were deeper interproximally than at buccal or palatal/lingual sites. This might explain more favorable PPD reduction at interproximal sites without adjusting for baseline PPD. Considering the different therapies, SRP and AF provided better PPD reduction than no therapy. However, the analysis failed to reveal statistically significant differences between both therapies in single-rooted teeth. It seems that AF provides no advantages over SRP in single-rooted teeth.

Taking radiographic parameters into consideration, current smoking and baseline PPD were kept in the models.
However, the greater the interproximal bone loss, the poorer the RAL-V gain $(p<0.0001)$, and infrabony defects were associated with inferior PPD reduction $(p<0.0001)$. Total amount of bone loss seems to be associated with poor prognosis of the respective tooth generally irrespective of the type of treatment. This has also been observed for longterm stability [17]. D'Aiuto et al. [5] did not assess radiographic parameters. However, they considered tooth mobility and found mobility was correlated with less favorable PPD reduction. It is interesting to note that interproximal grooves that had been assessed as root double contours failed to influence the outcomes of therapy. Up to our best knowledge, this is the first analysis on the influence of baseline bone loss on the outcome of nonsurgical or surgical periodontal therapy that does not use patient means but individual tooth parameters.

Within the limitations of the present study the following conclusions may be drawn: (1) Nonsurgical and surgical periodontal therapies are effective in single-rooted teeth; (2) severe interproximal bone loss and infrabony defects deteriorate the clinical results, and (3) there seem to be more defect-associated (tooth, site) factors influencing treatment outcome than patient-associated factors.

Acknowledgments This study was supported by Ivoclar Vivadent AG, Schaan, Liechtenstein. Further, we wish to thank James Turonis, LTC, Periodontics, US Army Dental Activity Heidelberg, Germany, and his wife Lynn, for their valuable and competent support in the preparation of this manuscript.

Table 11 Multilevel linear regression analysis — dependent variable: PPD reduction 6 months after therapy, $n=36$ patients, 1,796 sites

\begin{tabular}{lrrrr}
\hline & Estimate & SE & $d f$ & $F$ value \\
\hline Intercept & -2.0771 & 0.1364 & 34 & \\
Current smoking & -0.4646 & 0.1197 & 1,313 & 15.08 \\
Jaw (maxilla) & -0.1469 & 0.0433 & 1,313 & 11.51 \\
Baseline PPD & 0.6286 & 0.0119 & 1,313 & 0.0001 \\
Baseline Plaque Index & -0.0550 & 0.0332 & 1,313 & 0.0007 \\
Infrabony defect & -0.2932 & 0.0671 & 1,313 & $<0.0001$ \\
\hline
\end{tabular}




\section{References}

1. Badersten A, Nilveus R, Egelberg J (1981) Effect of nonsurgical periodontal therapy. I. Moderately advanced periodontitis. J Clin Periodontol 8:57-72

2. Badersten A, Nilveus R, Egelberg J (1984) Effect of nonsurgical periodontal therapy. II. Severely advanced periodontitis. J Clin Periodontol 11:63-76

3. Caffesse RG, Sweeney PL, Smith BA (1986) Scaling and root planing with and without periodontal flap surgery. J Clin Periodontol 13:205-210

4. Cohen ME, Ralls SA (1988) False positive rates in the determination of changes in probing depth related periodontal measurements. J Periodont Res 23:161-165

5. D'Aiuto F, Ready D, Parkar M, Tonetti MS (2005) Relative contribution of patient-, tooth-, and site-associated variability on the clinical outcomes of subgingival debridement. I. Probing depths. J Periodontol 76:398-405

6. Egelberg J, Claffey N (1994) Effect of initial periodontal treatment. In: Egelberg J (eds) (ed) In: Periodontal re-evaluation. The scientific way. Munksgaard, Copenhagen, pp 70-77

7. Eickholz P (1995) Reproducibility and validity of furcation measurements as related to class of furcation invasion. J Periodontol 66:984-989

8. Eickholz P, Kim T-S, Bürklin T, Schacher B, Renggli HH, Schaecken MT, Holle R, Kübler A, Ratka-Krüger P (2002) Nonsurgical periodontal therapy with adjunctive topical doxycycline: a double-blind randomized controlled multicenter study. I. Study design and clinical results. J Clin Periodontol 29:108-117

9. Fleischer HC, Mellonig JT, Brayer WK, Gray JL, Barnett JD (1989) Scaling and root planing efficacy in multirooted teeth. J Periodontol 60:402-409

10. Goldstein H (1995) Multilevel statistical models. Edward Arnold, London

11. Goldstein H, Browne W, Rasbash J (2002) Tutorial in biostatistics. Multilevel modelling of medical data. Stat Med 21:32153315

12. Goodson JM, Cugini MA, Kent RL, Armitage GC, Cobb CM, Fine D, Fritz ME, Green E, Imoberdorf MJ, Killoy WJ, Mendienta C, Niederman R, Offenbacher S, Taggert EJ, Tonetti M (1991) Multicenter evaluation of tetracycline fiber therapy. I. Experimental design, methods, and baseline data. J Periodont Res 26:361-370

13. Grossi SG, Zambon JJ, Ho AW, Koch G, Dunford RG, Machtei EE, Norderyd OM, Genco RJ (1994) Assessment of risk for periodontal disease. I. Risk indicators for attachment loss. J Periodontol 65:260-267

14. Heitz-Mayfield LJA, Trombelli L, Heitz F, Needleman I, Moles D (2002) A systematic review of the effect of surgical debridement vs non-surgical debridement for the treatment of chronic peri- odontitis. J Clin Periodontol 29(Suppl 3):92-102 discussion: $160-162$

15. Hung H-C, Douglass CW (2002) Meta-analysis of the effect of scaling and root planing, surgical treatment and antibiotic therapies on periodontal probing depth and attachment loss. J Clin Periodontol 29:975-986

16. Kaldahl WB, Kalkwarf KL, Patil D, Molvar MP, Dyer JK (1996) Long-term evaluation of periodontal therapy. I. Response to 4 therapeutic modalities. J Periodontol 67:93-102

17. Kaltschmitt J, Pretzl B, Eickholz P (2005) Langzeitergebnisse 10 Jahre nach parodontaler Therapie. 2. Zahnbezogene Faktoren. Dtsch Zahnärztl Z 60:211-214

18. Kim T-S, Bürklin T, Schacher B, Ratka-Krüger P, Schaecken MT, Renggli HH, Fiehn W, Eickholz P (2002) Pharmacokinetic profile of a locally administered doxycycline gel in crevicular fluid, blood, and saliva. J Periodontol 73:1285-1291

19. Kim C-K, Choi S-H, Kim T-S, Kaltschmitt J, Eickholz P (2006) The infrabony defect and its determinants. J Periodont Res 41:498-502 DOI 10.1111/j.1600-0765.2006.00895.x

20. Kim T-S, Klimpel H, Fiehn W, Eickholz P (2004) Comparison of the pharmacokinetic profiles of two locally administered doxycycline-gels in crevicular fluid and saliva. J Clin Periodontol 31:286-292 DOI 10.1111/j.1600-051X.2004.00494.x

21. Lindhe J, Socransky SS, Nyman S, Haffajee A, Westfeld E (1982) "Critical probing depth" in periodontal therapy. J Clin Periodontol 9:323-336

22. Löe H (1967) The Gingival Index, the Plaque Index and the Retention Index Systems. J Periodontol 38:610-616

23. Loos B, Nylund K, Claffey N, Egelberg J (1989) Clinical effects of rootdebridement in molar and non-molar teeth. A 2-year follow-up. J Clin Periodontol 16:498-504

24. O'Leary TJ, Drake RB, Naylor JE (1972) The plaque control record. J Periodontol 43:38

25. Rabbani GM, Ash MM Jr, Caffesse RG (1981) The effectiveness of subgingival scaling and root planing in calculus removal. J Periodontol 52:119-123

26. Ramfjord SP, Nissle RR (1974) The modified Widman flap. J Periodontol 45:601-607

27. Schei O, Waerhaug J, Lovdal A, Arno A (1959) Alveolar bone loss as related to oral hygiene and age. J Periodontol 30:7-16

28. Serino G, Rosling B, Ramberg P, Socransky SS, Lindhe J (2001) Initial outcome and long-term effect of surgical and non-surgical treatment of advanced periodontal disease. J Clin Periodontol 28:910-916

29. Tomar SL, Asma S (2000) Smoking-attributable periodontitis in the United States: findings from NHANES III. National Health and Nutrition Examination Survey. J Periodontol 71:743-751

30. Trombelli L, Cho K-S, Kim C-K, Scapoli C, Scabbia A (2003) Impaired healing response of periodontal furcation defects following flap debridement surgery in smokers. A controlled clinical trial. J Clin Periodontol 30:81-87 